\title{
Influence of Selected Exercise on Serum Immunoglobulin, Testosterone and Cortisol

\author{
in Semi-Endurance Elite Runners
}

Keyvan Hejazi* ${ }^{* 1}$, MSc; Seyyed-Reza Attarzadeh Hosseini ${ }^{2}$, PhD

\author{
Authors' Affiliation: \\ 1. Department of Sport Physiology, \\ Faculty of Physical Education and \\ Sport Sciences, Ferdowsi University \\ of Mashhad, Mashhad, Iran \\ 2. Faculty of Physical Education and \\ Sport Sciences, Ferdowsi University \\ of Mashhad, Mashhad, Iran \\ * Corresponding Author; \\ Address: Paradise Daneshgah, Azadi \\ Square, Faculty of Physical Education \\ and Sport Sciences, Ferdowsi \\ University of Mashhad, \\ PoBox: 1574, Mashhad, Iran \\ E-mail: keyvanhejazi@yahoo.com
}

Received: Feb 12, 2012

Accepted: Jun 05, 2012

Key Words: Immunoglobulin; Testosterone; Cortisol; Running; Athletes

\begin{abstract}
Purpose: The aim of this study was to compare the levels of serum immunoglobulin (IgA, IgM, IgG), testosterone and cortisol in semi-endurance elite runners during general preparation and competition phase of training.

Methods: Thirteen semi-endurance elite male runners with an average age of 18.92 \pm 1.7 years volunteered to take part in this study. The runners participated in the selected training for a period of 14 weeks and 12 sessions per week (in the morning and afternoon). Blood samples were collected during the three phases of training (before-preparation phase, after-preparation phase and beforecompetition phase). Data were analyzed by repeated measures and Bonferroni post hoc test, at a significance level of $P<0.05$.

Results: The levels of serum IgM in semi-endurance elite runners after preparation phase reduced significantly $(P=0.004)$, while these levels during the competition phase increased even though significantly. The levels of serum IgG and IgA also reduced, however not significantly, during both phases. Moreover, after preparation phase, there was no significant change in serum IgA levels; though, these levels reduced, however not significantly, before competition phase. Cortisol levels significantly decrease after preparation phase $(P=0.04)$; although, it increased before competition phase. Testosterone/cortisol ratio increases significantly after preparation phase $(P=0.04)$, and it decreased before competition phase. Testosterone levels intangibility increased and decreased respectively after preparation and before competition phases.
\end{abstract}

Conclusions: Findings indicated that long and intensive exercises weaken the immune system, while moderate and short drills strengthened this system.

Asian Journal of Sports Medicine, Volume 3 (Number 3), September 2012, Pages: 185-192

\section{INTRODUCTION}

Exercise can cause a change in the consistency of serum immunoglobulin (IgA, IgM, IgG) levels and secretion of the some hormones ${ }^{[1-2]}$. When athletes go under a lot of pressure, the levels of the immunoglobulin and hormones change; When athletes go under a lot of pressure, the amounts of their immunoglobulin and hormones change ${ }^{[1-2]}$, among these changes are significant variations in IgG, IgM, IgA, cortisol hormones and testosterone ${ }^{[3]}$.

Hanns et al reported that the amount of Serum IgG, IgA and IgM were increased within the first hours post- marathon and normalized in the recovery period. When changes in plasma volume were considered, only IgA increased significantly ${ }^{[4]}$. Andrew et al reported that a 60-minute downhill run on a treadmill (gradient $13.5 \%$ ) at a speed eliciting $75 \%$ of their VO2 peak on a level grade in 15 males caused the total of IgG, $\operatorname{IgG}_{1}$, $\mathrm{IgG}_{3}$, and IgA to be significantly higher and $\operatorname{IgG}_{2}$ significantly lower ${ }^{[5]}$. Imanipour et al in their study with active men, during 14 weeks found significant changes in IgA and IgM serum concentration, and there was no meaningful difference between pre and post test in IgG ${ }^{[6]}$. On the other hand, Verde et al reported that IgG, IgM concentration in ten elite male runners that 
participated in increased training schedules by an average of $38 \%$ for $3 \mathrm{wk}$, decreased significantly ${ }^{[7]}$.

Immunoglobulin plays an important role in protecting the body against infectious diseases ${ }^{[8-9]}$ and the increase in amounts of adrenaline, cortisol, growth hormone, and prolactin are among the changes which occur due to exercises, all of which have the a negative impact on immune system ${ }^{[10]}$. Among the abovementioned hormones, cortisol is a catabolism hormone which is secreted from the suoerficial layers of the adrenal gland and is considered an important factor in metabolism and immune system competence in the body $^{[11]}$.

According to the regular endurance exercise, cortisol (hypothalamic-pituitary-adrenal axis) and noradrenaline (norepinephrine) (sympathetic nervous system) have been shown to promote $B$ cell immunoglobulin isotope switching from IgM to $\operatorname{IgG}^{[1]}$. Djken and colleague stated that intense physical training led to increased serum cortisol and IgA, IgM, IgG in active and none active people ${ }^{[3]}$. On the other hand, Mackinnon et al reported that short moderate training caused serum IgA and IgM decrease significantly without variation in levels of IgG ${ }^{[12]}$.

These changes in the production of immunoglobulins have been related to the stimulation of the central nervous system and the increase of catecholamine. Therefore, it can be inferred that the differences among subjects can be attributed to their adaptation to exercise ${ }^{[13]}$. While, cortisol has a catabolic effect, testosterone is responsible for the stimulation of the anabolic process of skeletal muscle growth increase linearly in response to exercise ${ }^{[14]}$. In this case, Kaye and colleagues reported that there was not a significant connection among the total amounts of testosterone and cortisol, or free testosterone and cortisol during the rest time. However, there is a significant connection in recycling time after exercising among these items. They stated that an exercise with an intensity of $65 \%$ to $75 \%$ of oxygen consumption causes a negative significant connection between cortisol and free testosterone ${ }^{[15]}$. Hazar and colleagues reported that after doing some maximum aerobic exercise, there was a noticeable increase in the levels of testosterone; while they could not spot a big difference in cortisol levels ${ }^{[16]}$.
In summary, considering the importance of stress and its role in sports competitions, an understanding of physiological and psychological conditions during competitions is very crucial, the lack of studies on effects of demanding, and constant exercises in warmup and bodybuilding of athletes, esp. half marathon runners, on serum immunoglobulin, testosterone and cortisol are among factors which have made researchers examine the fluctuations in the amount of serum immunoglobulin IgA, IgM, IgG, testosterone and cortisol in male semi-endurance runners during the preparation and competition phases.

\section{METHODS AND SUBJECTS}

\section{Participants:}

This research was semi-experimental with three phases which were as follows: before preparative phase, after preparative phase and before competitive phase in one experimental group. Subject samples of this research have been selected runners from the semi-endurance Iranian national team who were professionally practicing this sports discipline for $4.84 \pm 0.80$ years. Thirteen national level male runners undergoing training in the Sports Authority of Iran were selected voluntarily and objectively participated in this study. During the first stage, individuals were introduced to the concept and ways of co-operating. Important notes were given on athletic exercises, nutrition, diseases, drug consumption, supplements, energy drinks, and drug abuse so that they could take necessary measures to consider them. Then, in order to make sure that none of runners were carrying any of the following diseases including the common cold, influenza, sore throat, coronary sweating, diabetes, kidney and thyroid malfunction, they filled up a self-testifying questionnaire, and after the approval of its standards, the participation and co-operation agreement form, which allowed them to be the subjects of the study, was signed by the qualified runners. During the second stage, their height was measured in centimeters using a height determiner and their weight was calculated using a digital scale produced by a German company 
called Beurer (PS07-PS06). Then, the waist-hip ratio was determined. Body fat percentage was calculated using a body compound determiner (model In-body720 made in Korea) and based on a method called bioelectrical impedance. All of these measurements were carried out while the runners had stopped eating or drinking 4 hours prior to their test, and their bladder, stomach, and bowels were totally empty.

\section{Biochemical tests:}

The serum levels IgG, IgM, IgA, cortisol and testosterone of all runners were measured at three points in time: a) immediately prior to the study, b) at the end of the preparation period, c) at the before the competition period, amount of 10 milliliters of blood was taken from athletes' right-elbow vein between 15:00 to $16: 00$ in three stages in the research: which was a five-weeks preparation period and nine-weeks competition period.

The runners were asked not to get involved in any serious or intense physical activity three days prior to their blood sample extraction. The plasma of their blood sample was immediately separated from their blood and was frozen in laboratories in -20 degrees Celsius. In order to measure serum immunoglobulin types IgG, IgM, IgA automatically, a nephelometry was used, and a Binding Site kit (made in the U.K.) was also used. Moreover, immunoradiometric Assay by means of a Gamma Counter method was used for the quantitative estimation of testosterone and cortisol (RIA Kits from Omega diagnostics, France).

To make athletes have similar nutrition, which could have an impact on study elements; subjects were required to write down a checklist report for their three-day diet. Therefore, after collecting all the nutrition information, the amount of received calorie and supplements was distinctly clear. In addition to that, the subjects were asked to consume a common type of food with the same calorie value prior to two periods of blood testing.

\section{Exercise Programs:}

The selected exercise program in this study was the same as the advanced coordinated program designed for semi-endurance male runners by the government, which was carried out every morning and afternoon, six days a week, and for 14 weeks.

The selected training during preparation phase included: the morning exercise consisting of warm-up, flexibility, the basic moves for running tracks; and aerobic exercises with 40-60 percent of maximum heart rate reserve (MHRR). Weight training program was made up of Squat, front thighs, back thighs, standing shin, sitting shin, chest press, shoulder in three set(s) and every set was 12 repeated considering 50 percent one repetition maximum and 2-min rest between every set(s). The afternoon exercise included: A) warm-up exercises, Fartlek running with 1000 to $6000 \mathrm{~m}$ with $60 \%$ MHRR, interval training with 1 to 2 repetitions in 200 to $1200 \mathrm{~m}$ with fast and even steps along with 75 percent of maximum speed, slow but steady marathon running, and or B) aerobic running for 8000 to 14000 m with 60-70\% MHRR, unsteady, accelerated speed running and slope running. Furthermore, training during competition phase consisted: the morning exercise consisted of warm-up, flexibility, the basic moves for running tracks, and aerobic exercises with 55-65 percent of MHRR. Weight training program was made up of Squat, front thighs, back thighs, standing shin, sitting shin, chest press, shoulder in three set(s) and every set was 8-10 repeated considering 60 percent one repetition maximum and 1.5-min rest between every set(s). Special training such as plyometric exercise with 50 percent of MHRR. The afternoon exercise included: lactate acid training (short and long interval) with 3000 to $8000 \mathrm{~m}$ with 70-90 MHRR. Aerobic running for 6000 to $12000 \mathrm{~m}$ with $60-80 \%$ MHRR, Speed running with 2000 to 3000m with 8090\% MHRR, Fartlek's running with 6000 to $12000 \mathrm{~m}$ with $60-70 \%$ MHRR and performed slope running with 3000 to $4000 \mathrm{~m}$ with $70 \% \mathrm{MHRR}$.

The MHRR for every single athlete was respectively calculated based on the Karvonen equation (1) and was also controlled during exercise by a heart rate monitor (made in Finland-Polar), and maximum repetition was determined using equation (2) ${ }^{[17-18]}$.

Equation (1): Target heart rate= [\%60-\%70+ [(resting pulse(age-220)]] + Resting heart rate

Equation (2): One maximum repetition = displaced weight (kilograms)/ (0.0278 x number of repetition to exhaustion) 1.0278 
Table 1: General characteristics of participants ( $\mathrm{N}=13)$

\begin{tabular}{|lc}
\hline Variables & Mean (standard deviation) \\
Age (years) & $18.92(1.7)$ \\
Height $(\mathbf{c m})$ & $177.69(5.32)$ \\
Weight $\mathbf{( k g )}$ & $63.49(6.65)$ \\
Body mass index (kg/m2) & $20.07(1.50)$ \\
Subjects background in sports (yr) & $4.84(0.80)$
\end{tabular}

\section{Statistical analysis:}

Finally, all the data were analyzed using SPSS version calculated after checking the data distribution normalcy using Kolmogorov-Smirnov test and Homogeneity of variance method and then examined by repeated measures and Bonferroni post hoc test, at a significance level of $P<0.05$.

\section{RESULTS}

Table 1 shows means for age, height, weight, BMI and subject's background in sports. The average, standard deviation and results coming from within the group's changes of levels IgG, IgM, IgA, testosterone, cortisol and testosterone/cortisol ratio ( $\mathrm{T} / \mathrm{C}$ ratio) of selected runners is presented in table 2.

According to table 2, the fitness training program had a significant impact on elite runners’ weights and

Body Mass Index $(P<0.05)$ in a way that during the Fitness training program, 1.7 percent of body weight and 2.1 percent of Body Mass Index suffered reduction. During preparation period, body fat was reduced from 10.05 percent to 9.61 percent, which is not significant. Likewise, weights and Body Mass Index during competition phase, decrease significantly $(P<0.05)$.

Despite the fact that the IgG level of male semimarathon runners decreased by 6.63 percent during the preparation period, this change was not significant.

The in-group IgM level average variance of selected male semi-marathon runners had a significant reduction during preparation period $(P<0.05)$. This reduction was around 27.66 percent. However, this level increased during competition phase. Furthermore, there has not been a meaningful change in IgA levels of runners during the preparation period .So it has decreased by $5.75 \%$ during competition phase; this change was not significant. Fig. 1 shows the fluctuations in IgM, IgG, IgA, testosterone, cortisol and testosterone/cortisol ratio levels of male semimarathon runners in preparation and before

Table 2: Mean of variation of within groups anthropometrics, serum IgA, IgM, IgG, testosterone cortisol and T/C ratio in elite runners $(\mathrm{N}=13)$

\begin{tabular}{lccccc} 
Variable & $\begin{array}{c}\text { Pefore } \\
\text { preparation* }\end{array}$ & $\begin{array}{c}\text { Phases } \\
\text { After } \\
\text { Preparation* }\end{array}$ & $\begin{array}{c}\text { Vefore } \\
\text { competition* }\end{array}$ & \multicolumn{2}{c}{$\begin{array}{c}\text { Variation of within } \\
\text { groups }\end{array}$} \\
\hline Weight (kg) & $63.49(6.65)$ & $62.41(6.43)$ & $61.58(6.24)$ & 7.41 & 0.003 \\
Body mass index (kg/m²) & $20.07(1.50)$ & $19.65(1.44)$ & $19.39(1.61)$ & 15.40 & 0.000 \\
Body fat percent (\%) & $10.05(2.91)$ & $9.61(2.21)$ & $9.53(1.59)$ & 0.38 & 0.689 \\
IgG (mg/dl) & $1199.5(316)$ & $1120.0(277.0)$ & $1026.8(359.0)$ & 1.04 & 0.368 \\
IgM (mg/dl) & $112.8(30.2)$ & $81.6(35.1)$ & $102.2(19.9)$ & 6.90 & 0.004 \\
IgA (mg/d) & $168.3(64.3)$ & $168.1(62.2)$ & $162.4(58.2)$ & 0.32 & 0.726 \\
Cortisol (nmol/L) & $299.7(97.7)$ & $219.1(71.6)$ & $233.9(99.0)$ & 3.65 & 0.041 \\
Testosterone (nmol/L) & $5.84(2.42)$ & $6.13(2.45)$ & $4.82(2.14)$ & 1.81 & 0.185 \\
T/C ratio (nmol/L) & $0.0194(0.008)$ & $0.028(0.015)$ & $0.021(0.011)$ & 3.69 & 0.040 \\
$\quad *$ Values are mean (standard deviation). & & & & &
\end{tabular}




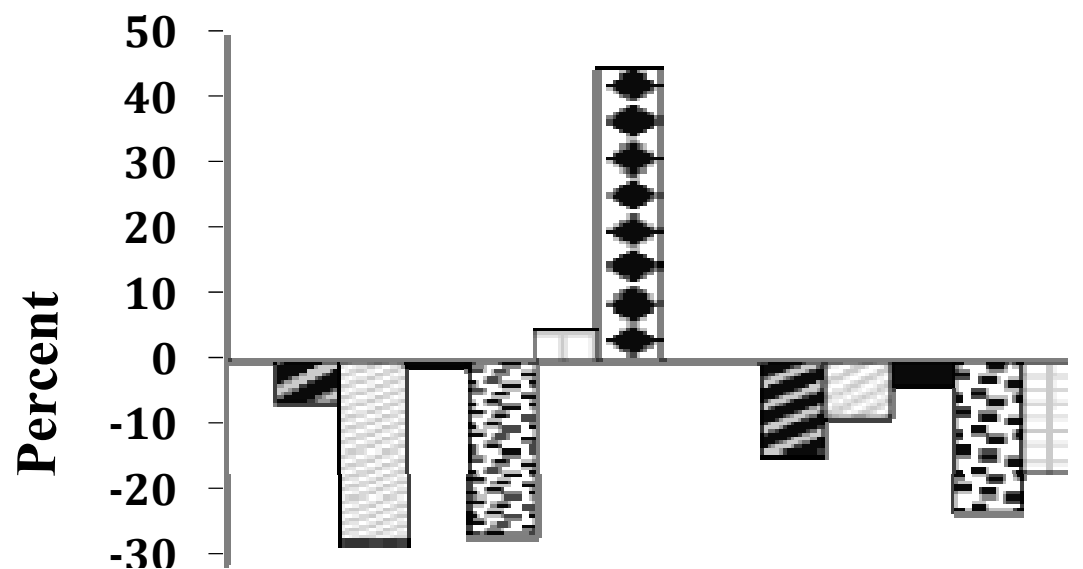

Fig. 1: Percent of variation of serum IgA, IgM, IgG, testosterone, cortisol and T/C in semi-endurance runners during preparation and competition phases compared to before the preparation phases

competition phase than amount of these before the preparation period. According to the fig. 1, serum IgA, IgM, IgG and cortisol in runners had a significant reduction during preparation and before competition period than the amount before the preparation period.

The level of the testosterone had increased in preparation period and during competition period compared to before the preparation period decreased. Testosterone/cortisol ratio in both of the preparation and competition period increased than before the preparation period.

\section{DISCUSSION}

In this study, 84 sessions of workout had a meaningful impact on body weight and body mass index. Interestingly, body weight is something that most researchers expect to be changed during body-building exercises; however, according to the analysis of studies, the quality of body weight changes and how it happens during body-building workout is different ${ }^{[19]}$.

In this research, It seems that one of the main reasons in losing body weight and body fat mass is the calorie limitation and the change in the length of the track used by runners. In addition to that, the change in body weight could be caused by a change in runners' psychological state ${ }^{[19]}$.
According to this research IgA intensity stayed the same before and after the preparation period, while it was $5.75 \%$ during competition period, which was not significant.

Regardless of the fact that IgA is responsible for membranous immunity and much of that is available in human saliva, IgM and IgG play an important role in humoral immunity ${ }^{[20]}$. Immunoglobulin $\mathrm{G}$ is one of the most important serum immunoglobulins and its smooth, natural function is of great weight for human immune system, and it also protects our bodies from infections and other germ activities ${ }^{[21]}$.

According to this research, 84 sessions of exercise during preparation time did not have a significant meaning on serum IgG in athletes' rest time, Hence, this level has reduce during competition period, even so, it was not significant. Imanipour et al, in their study with active men, during 14 weeks found no significant difference between pre and posttests in IgG ${ }^{[6]}$. Verde et al reported that IgG concentration in ten elite male runners that participated in increased training schedules by an average of 38\% for 3 wk, decreased significantly ${ }^{[7]}$.

The current study results about immunoglobulin $\mathrm{M}$ show that preparation period exercises brought about $27.66 \%$ decrease in IgM, during competition period IgM reduced more than before the preparation period, while it was not significant.

Mckune and colleagues reported a significant decrease by 23 percent in IgM intensity after a 24-hour 
full marathon ${ }^{[1]}$. Coffman and colleagues confirmed IgM reduction after the marathon ${ }^{[22]}$. There is this possibility that the changes in serum immunoglobulins depend on the intensity and the time of exercise.

Overall, the researchers have concluded that the changes in immunoglobulin intensity depend on several factors; thus, they suggested different mechanisms to justify the Ig intensity changes a few of which we will mention here ${ }^{[21]}$.

Ig level has to be calculated while examining plasma volume changes. The slight increase in Ig intensity (20 percent), which happens after vigorous exercises, is somehow related to plasma volume changes. The less than 10 percent increase in Ig intensity is usually caused by the daily changes or exchanges of outer veins, lymph vessels, or the blood circulation. Therefore, the decreasing 27, 23, 20 to 35 percent changes in of IgM volume in this study and other studies cannot be based on plasma volume changes ${ }^{[1,7,23]}$.

Although most of the contrasting studies indicate that immunoglobulin level and its natural amount in resting athletes do not differ from that of non-athletes, the Ig intensity and antibody production decreased in those runners and athletes who practiced intense and competitive training ${ }^{[20]}$.

According to this research, cortisol levels significantly decrease during preparation phase; although, it increased during pre-competition phase. testosterone levels intangibility increased and decreased respectively during preparation and precompetition phases. T/C ratio increases significantly during preparation phase and it decreased during precompetition phase.

Thus, these findings correspond with findings from Lehmann and colleagues (1992), who reported the decrease in cortisol without variation in testosterone after 3 weeks by an increase in the volume and intensity during training in endurance and semiendurance runners ${ }^{[24]}$. Daly and colleagues (2005) after studying the effect of prolonged endurance exercise reported a significant reduction in cortisol in athletes ${ }^{[25]}$. There have been several reasons for changes in intensity of cortisol in body after physical activities, including hypothalamus stimulation, Pituitary, Adrenal (HPA), adrenocorticotropic release, central body temperature, PH changes, sympathetic nervous system, hypoxia, and ethacridine lactate gathering. Vigorous body activity with 60 to 65 percent of oxygen consumption stimulates HPA axis and increases core temperature; it also causes an increase in releasing cortisol and freeing them from carrying proteins. The increase in pituitary response during vigorous exercises has a big impact on androgenic anabolic. ${ }^{[26]}$.

According to the conducted research, the amount of cortisol release is different during days and the most amounts are released during mornings, and the closer we get to the afternoons the less the amounts of that is seen, except in stress periods in which its level does not increase ${ }^{[27]}$, Since the time of collecting samples was in the afternoon and the subjects were in the recess time without having any stress, these factors might have influenced the release of cortisol slightly. Adaptation index to exercises in males is used through considering the ratio of free testosterone to cortisol and the ratio of testosterone to cortisol ${ }^{[28]}$.

An increase in the ratio of free testosterone to cortisol is mainly caused by a decrease in serum cortisol levels. A decrease of cortisol after exercise is probably due to an increase in cortisol circulation removal, or adrenocorticotropic hormone decrease ${ }^{[29]}$. In the period before the competitions, the release of anabolic and catabolic hormones has been reverted in a way that cortisol levels increased more than preparation period. At this period, the ratio of serum testosterone and testosterone to cortisol ratio had decreased, which could indicate an increase in the catabolic procedure of runners' bodies before the competitions. The result of the research shows that whenever T/C ratio decreases more than 30 percent and this situation continues to prolong, it can lead to overtraining for the athletes ${ }^{[30]}$.

\section{CONCLUSION}

According to the conducted studies, it has been determined that body activities are one of the effective factors in changing of immune system process, which depends on the intensity, time, training plan, and body 
athleticism. The findings in this study indicated that the five-week preparation training and the nine-week competition training in 6 days, 2 evening and morning sessions (84 sessions) does not affect the humoral immunity (IgG, IgA); however, this does affect IgM and cortisol significantly. With respect to different study results, researchers believe that long, vigorous exercise weakens the immune system, and mild short exercises will strengthen the immune system. Overall, we can conclude that physical exercise in halfmarathon runners will result in the weakening of the immune system. Therefore, trainers and physicians are advised to consider factors which will strengthen the immune system while planning vigorous and intense exercise.

\section{ACKNOWLEDGMENTS}

This study was funded by the Research department of Mashhad University; we gratefully acknowledge them and thank the athletes and Hashem Siyami the track and field coacher in Khorasan Razavi for the invaluable help they offered. The project was approved by the Ethics Committee for Scientific Research at the Academy of Physical Education under code of 286312 in Ferdowsi University of Mashhad, IRAN.

\section{Conflict of interests: None}

\section{REFERENCES}

1. Mckune A, Smith L, Semple S, Wadee A. Influence of ultra-endurance exercise on immunoglobulin isotypes and subclasses. Br $J$ Sports Med 2005;9:665-70.

2. Dimitriou L, Doherty M. Circadian effects on the acute responses of salivary cortisol and IgA in well trained swimmers. Br J Sports Med 2002;36:260-4.

3. Djken H, Kelle M, Colpan L, et al. Effect of physical exercise on complement and immunoglobulin levels in wrestlers and sedentary controls. J Med School 2000;27:39-45.

4. Hanns G, Machotta A, Schobersberger W, et al. Neopterin, IgG, IgA, IgM, and plasma volume changes during long-distance running. Pteridines 2002;13:15-20.

5. Andrew M, Smith L, Wadee A. Complement, immunoglobulin and creatine kinase response in black and white males after muscledamaging exercise. SAJSM 2009;21:47-52.

6. Imanipour V, Shahedi V, Armandnia M. The effects of physical activity on homoral immune system (IgA, IgG, IgM). Procedia Soc Behav Sci 2009;1:2718-21.

7. Verde T, Thomas S, Moore R, et al. Immune responses and increased training of the elite athlete. J Applied Physiol 2007;103:14-5.

8. Alexander J, Koch. Immune response to resistance exercise. Am J Lifestyle Med 2010;11:244-52.

9. Laing S, Gwynne D, Blackwell J, et al. Salivary Iga response to prolonged exercise in a hot environment in trained cyclists. Eur $J$ Appl Physio 2005;93:665-71.

10. Maughan R, Gleeson M, Greenhaff P. Biochemistry of exercise and training. 1ed. USA: Oxford University; 1997.

11. Mcguigan M, Egan A, Foster C. Salivary cortisol responses and perceived exertion during high intensity and low intensity bouts of resistance exercise. J Sport Sci Med 2004;3:8-15.

12. Mackinnon L, Ginn E, Seymour G. Temporal relationship between decreased salivary IgA and upper respiratory tract infection in elite athletes. Aust J Sci Med Sport 1993;25:94-9.

13. Karacabey K, Peker I, Saygin O, et al. Effect of acute aerobic and anaerobic exercise on humoral immune factors in elite athletes. Biotechnol Biotechnol 2005;19:175-80.

14. Thomas N, Leyshon A, Hughes $\mathrm{M}$, et al. The effect of anaerobic exercise on salivary cortisol, testosterone and immunoglobulin (A) in boys aged 15-16 years. Eur J Appl Physiol 2009;107:455-61.

15. Kaye K, Alex W, Moore AW, Hackney AC. Relationship between circulation cortisol and testosterone: influence of physical exercise. J Sports Sci Med 2005;4:76-83.

16. Hazar S, Hazar M, Korkmaz S, et al. The effect of graded maximal aerobic exercise on some metabolic hormones, muscle damage and some metabolic end products in sportsmen. Sci Res Essays 2011;6:1337-43.

17. Robbert A, Landwehr R. The supporting history of the "HRmax=220-age" equation. $J$ Exer Physiol Online 2002;5:1-10. 
18. Lee E, Brown Joseph P, Weir. ASEP procedures recommendation I: accurate assessment of muscular strength and power. $J$ Exer Physiol Online 2001;4:1-2.

19. Paul T, Thompson W. Dose-dependent effects of training and detraining on weight in 6406 runners during 7.4 years. Obesity 2006;14:1975-84.

20. Neil C, Gleeson M, MacLaren D. Immune function in sport and exercise: Advances in Sport and Exercise Science Series. $1^{\text {st }}$ ed. New York: Churchill Livingstone. 2006; Pp:115-8.

21. Laurel T. Advance in exercise immunology. $1^{\text {st }}$ ed. Cincinnati, Ohio: Human kinetics; 1999.

22. Coffman RL, Lebman DA, Rothman P. Mechanism and regulation of immunoglobulin isotype switching, Adv Immuno 1993;54: 22970.

23. Byum A, Wiik B, Gustavsson E, et al. The effect of strenuous exercise, calorie deficiency and sleep deprivation on white blood cells, plasma immunoglobulin and cytokines. Scand J Immunol 1996;43:228-35.

24. Lehmann M, Gastmann M, Petersen M, Bachl M, Seidel C, Med A, et al. Training - overtraining: performance, and hormone levels, after a defined increase in training volume versus intensity in experienced middle- and long-distance runners. $\mathrm{Br} J \mathrm{Sp} \mathrm{Med}$ 1992;26:233-42.

25. Daly W, Seeqers C, Rubin D, Hackney A. Relationship between stress hormones and testosterone with prolonged endurance exercise. Eur J Appl Physiol 2005;93:375-89.

26. Jankord R, Ganjam V, Turk J, Hamilton M, Laughlin M. Exercise training alters effect of high-fat feeding on the ACTH stress response in pigs. Appl Physiol Nutr Metab 2008;33:461-9.

27. Kakooei H, Zamanian Ardakani Z, Karimian M, Ayttollahi $\mathrm{T}$.Twenty four hours circadian cortisol profile in shift work nurses. Armaghan Danesh J 2009;14:47-56. (In Persian)

28. Mujika I, Chatard J, Padilla S. Hormonal responses to training and its tapering off in competitive swimmers. Eur J Appl Physiol Occup Physiol 1996;74:361-6.

29. Heshmat H, Moussa A, Emara O. Estimation of the integrity of vital organs and the balance between catabolism and anabolism in athletes and non-athletes. World J Sport Sci 2010;3:S668-72.

30. Majumdar P, Srividhya S ,Mandal M, Kalinski M. Response of selected hormonal markers during training cycles on Indian female swimmers. Biol Sport 2010;27:53-7. 